\title{
FOXL1 overexpression is associated with poor outcome in patients with glioma
}

\author{
AINIAN CHEN ${ }^{1}$, LINGLING ZHONG ${ }^{1}$ and JIA LV $^{2}$ \\ Departments of ${ }^{1}$ Neurology and ${ }^{2}$ Neurosurgery, Huai'an First People's Hospital, \\ Nanjing Medical University, Huai'an, Jiangsu 223300, P.R. China
}

Received December 19, 2018; Accepted April 19, 2019

DOI: $10.3892 /$ ol.2019.10351

\begin{abstract}
Gliomas are the most common primary tumors in adult central nervous system and result in disappointing survival outcomes. FOXL1, as a transcription factor, plays an important role in regulating the expression of genes involved in cell metabolism, proliferation and differentiation. In this study, we investigated the relationship between FOXL1 expression and prognosis of patients with glioma. We selected 611 glioma patients from The Cancer Genome Atlas (TCGA) database and 132 glioma patients from Huai'an First People's Hospital (PFHH). The prognostic values of FOXL1 in glioma were analyzed in both cohorts. In TCGA cohort, the median (10.2389) was used as the cut-off value of FOXL1 mRNA levels in tumor tissue. Kaplan-Meier analysis showed that higher WHO glioma grade $(\mathrm{P}<0.001)$ and expression of FOXL1 $(\mathrm{P}<0.001)$ were associated with worse overall survival (OS). The univariate Cox regression model revealed that age $(\mathrm{P}<0.001)$, WHO grade $(\mathrm{P}<0.001)$, histological type $(\mathrm{P}<0.001)$ and FOXL1 expression $(\mathrm{P}<0.001)$ were associated with prognosis of glioma patients. In PFHH cohort, expression of FOXL1 in tumor cells was detected by immunohistochemistry (IHC) staining based on a tissue microarray (TMA) sample. Kaplan-Meier analysis also showed that WHO glioma grade $(\mathrm{P}<0.001)$ and expression of FOXL1 $(\mathrm{P}=0.012)$ were associated with $\mathrm{OS}$ in glioma patients. The univariate Cox regression showed that $\mathrm{WHO}$ grade $(\mathrm{P}=0.001)$, histological type $(\mathrm{P}<0.001)$ and FOXL1 expression $(\mathrm{P}=0.013)$ were associated with prognosis of glioma patients. In both cohorts Kaplan-Meier subgroup analyses showed FOXL expression correlated with OS in high WHO grade subgroup, while low grade subgroup showed no such correlation. This study showed that higher expression of FOXL1 is associated with poor OS of glioma patients in TCGA and PFHH cohorts. Especially,
\end{abstract}

Correspondence to: Dr Ainian Chen, Department of Neurology, Huai'an First People's Hospital, Nanjing Medical University, 6 Beijing Road West, Huai'an, Jiangsu 223300, P.R. China

E-mail: zuid325@163.com

Key words: glioma, FOXL1, The Cancer Genome Atlas, outcome
FOXL1 overexpression is associated with worse outcomes in high WHO grade subgroup. Our findings suggest that FOXL1 expression is a candidate predictor of clinical outcome in glioma patients and may act as an effective molecular marker for immunotherapeutic strategies of glioma patients in clinical practice.

\section{Introduction}

Gliomas, which arise from glial cells, make up approximately $30 \%$ of all brain and central nervous system tumors and $80 \%$ of all malignant brain tumors (1). According to the WHO pathologic grading system, which is the most common of numerous grading systems in use, gliomas are further categorized as four grades (I-IV) (2). Gliomas with lower grade indicate better prognosis while higher grade gliomas indicate worse prognosis and increased fatality (3). At present, the standard treatment for gliomas includes maximal surgical resection and concurrent chemo-radiotherapy. However, the prognosis of glioma is still poor. The survival time of glioblastoma multiform (GBM, grade IV) patients is only approximately one year (4), and for grade II and III gliomas, the survival time is 2 and 2-5 years, respectively (5). Therefore, it is of great importance to find more effective molecular prognostic markers for the treatment of glioma patients in clinical practice.

FOX (Forkhead box) proteins are a super family of transcription factors that play crucial roles in regulating the expression of genes involved in cell metabolism, proliferation, differentiation and apoptosis (6). Many FOX proteins are at the junction of multiple signaling pathways, thus are important to embryonic development (7). Due to the vital roles in growth and development, the malfunctions of FOX proteins play important roles in a variety of pathological processes including cancer. For instance, FOXOs can initiate apoptosis and cause cell cycle arrest (8) and increase.

FOXM1 gene expression is often found in various human cancers (9). In the context of FOXL1 protein, it was first discovered in the mesenchyme of the gastrointestinal tract (10) and it played an important role in gut maintenance (11). Thus far, several studies have reported the associations of FOXL1 with gastrointestinal cancer including stomach, colon and pancreas (12-14), and urinary cancer $(10,15)$. In addition, Nakada et al reported that FOXL1 could regulate centralnervous system development by suppressing Sonic Hedgehog 
protein expression in zebrafish (16), suggesting that FOXL1 may be also involved in brain cancer.

This study investigated the expression of FOXL1 by immunohistochemistry (IHC) on a tissue microarray (TMA) including 132 glioma specimens in Huai'an First People's Hospital (Huai'an, China) and investigated its association with the survival outcome. Besides, 611 glioma patients from The Cancer Genome Atlas (TCGA) database were also included. To our knowledge, this is the first study to investigate the prognostic value of FOXL1 in glioma patients.

\section{Patients and methods}

TCGA database. The FOXL1 expression and clinical data in glioma patients of TCGA database were downloaded from the TCGA Research Network: http://cancergenome.nih.gov/. According to parameters defined in previous studies $(17,18)$, 611 glioma patients with detailed FOXL1 RNA seq information, fully characterized tumors, intact overall survival (OS) were included in our study. Clinicopathological characteristics, including age, sex, historical type and WHO grade were collected.

Patients from Huai'an First People's Hospital (PFHH). The TMA used for this study includes 132 unselected, non-consecutive, primary, and sporadic gliomas treated between March 2009 and August 2015 in Department of Neurosurgery, Huai'an First People's Hospital. Formalin-fixed, paraffin-embedded tissue blocks from resected glioma were made. Tissue cylinders with a $2.0 \mathrm{~mm}$ diameter were punched from representative tissue areas. The histological types were confirmed by experienced pathologists. The TMAs contained well-documented demographic and clinicopathological information, including patients' age, sex, WHO grade, histology types.

This study was approved by the Ethics and Research Committees of Huai'an First People's Hospital, (Project identification code: IRB-KPJ2017-003-01). Patients who participated in this research had complete clinical data. The signed informed consents were obtained from the patients or the guardians.

Immunohistochemical staining. IHC was performed according to the standard streptavidin-peroxidase (S-P) method (Zymed, San Francisco, CA, USA). The tissue wes fixed with $4 \%$ formaldehyde at $22^{\circ} \mathrm{C}$ for $12 \mathrm{~h}$ and then embedded with paraffin. The thickness of sections was $3 \mu \mathrm{m}$. Briefly, in a xylene and alcohol bath solution TMAs were dewaxed and dehydrated. Heat mediated antigen retrieval was performed with Tris/EDTA buffer $\mathrm{pH}$ 9.0. The endogenous peroxidase activity was then blocked using $0.3 \%$ hydrogen peroxide for $10 \mathrm{~min}$ at $22^{\circ} \mathrm{C}$. At room temperature the slides were cooled and blocked by incubating with normal goat serum for $1 \mathrm{~h}$. After that, the slides were subsequently incubated overnight at $4^{\circ} \mathrm{C}$ with rabbit anti-human FOXL1 polyclonal antibody (cat. no. ab190226; dil, 1:500; Abcam, Cambridge, UK). The sections were next incubated with biotinylated secondary goat anti-rabbit polyclonal antibody (cat. no. ab6720; dil, 1:800; Abcam, Cambridge, UK) for $30 \mathrm{~min}$ at room temperature, followed by incubation with streptavidin horseradish peroxidase complex. Finally, sections were visualized by 3,3'-diaminobenzidine staining. Then the slides were stained with hematoxylin and eosin (H\&E).

Scoring of IHC. The immunostaining signals were evaluated with microscope (Olympus, Tokyo, Japan) by two experienced pathologists who were blinded to the patients' clinical and pathological features. FOXL1 expression was scored according to staining intensity and the percentage of positive cells as described before (19). Briefly, the staining intensity was scored as 0 (negative), 1 (weak), 2 (medium) or 3 (strong). The percentage of positive cells was scored as follows: $<5 \%$ (0), 5-25\% (1), 25-50\% (2), 50-75\% (3) and 75-100\% (4) according to the percentages of the positive staining areas in relation to the whole glioma area. Scores for staining intensity and percentage positivity of cells were multiplied to generate the immune-reactivity score (IRS) for each case. Samples with a final staining score of $\leq 6$ were classified as low expression, while those with score of $>6$ were considered to be high expression.

Statistical analysis. All statistical analyses were carried out by the SPSS v.16.0 (SPSS, Inc., Chicago, IL, USA). Chi-square test and Fisher's exact test were used to analyze the association between clinicopathological parameters and FOXL1 expression. OS was defined as the interval from date of diagnosis until death from any cause. Data were censored for living patients and patients lost between follow-ups. Survival analysis was performed using the Kaplan-Meier method with log rank test and Cox regression model. All confidence intervals (CIs) were stated at the $95 \%$ confidence level. All statistical tests were two sided. $\mathrm{P}<0.05$ was considered to indicate a statistically significant difference.

\section{Results}

Clinical characteristics of glioma patients in TCGA cohort and PFHH cohort. In the TCGA cohort, the age of the 611 glioma patients ranged from 14 to 87 years, with a median value of 46 . Of the patients, 256 (41.9\%) were females and 355 (58.1\%) were males. The median follow-up time was 13.4 months and 182 patients died during follow-up. In the PFHH cohort, the age of 132 glioma patients ranged from 7 to 75 years, with a median value of 51 years. Of the total $56(42.4 \%)$ were females and $76(57.6 \%)$ were males. The median follow-up time was 10.0 months and 50 patients died during follow-up. Table I showed the relationship between FOXL1 expression and clinicopathological features of glioma patients in both cohorts. In the cohort of TCGA, FOXL1 expression was associated with age $(\mathrm{P}<0.001)$, WHO grade $(\mathrm{P}<0.001)$ and histological type $(\mathrm{P}<0.001)$. In the $\mathrm{PFHH}$ cohort there were no significant associations found between FOXL1 expression and clinicopathological features (Table I).

Expression pattern of FOXL1 in TCGA and PFHH cohorts. In the TCGA cohort, the median (10.2389) was used as the cut-off value of FOXL1 mRNA levels in tumor tissue of eligible patients. Patients were divided into low FOXL1 and high FOXL1 groups for further analysis (for low FOXL1 group, median: 6.2389, IQR: 3.54; for high FOXL1 group, 
Table I. Clinical characteristics of glioma patients in TCGA cohort and PFHH cohort.

\begin{tabular}{|c|c|c|c|c|}
\hline \multicolumn{5}{|l|}{ A, TCGA } \\
\hline \multirow[b]{2}{*}{ Variables } & \multirow[b]{2}{*}{ Cases, no. (\%) } & \multicolumn{2}{|c|}{ FOXL1 } & \multirow[b]{2}{*}{ P-value } \\
\hline & & Low & High & \\
\hline Age (years) & & & & $<0.001$ \\
\hline$<60$ & $471(77.1)$ & $256(85.3)$ & $215(69.1)$ & \\
\hline$\geq 60$ & $140(22.9)$ & $44(14.7)$ & $96(30.9)$ & \\
\hline Sex & & & & 0.909 \\
\hline Female & 256 (41.9) & $125(41.7)$ & $131(42.1)$ & \\
\hline Male & $355(58.1)$ & $175(58.3)$ & $180(57.9)$ & \\
\hline WHO grade & & & & $<0.001$ \\
\hline Low & $222(36.3)$ & $155(51.7)$ & $67(21.5)$ & \\
\hline High & $389(63.7)$ & $145(48.3)$ & $244(78.5)$ & \\
\hline Histological type & & & & $<0.001$ \\
\hline Oligoastrocytoma & $117(19.1)$ & $74(24.7)$ & $43(13.8)$ & \\
\hline Oligodendroglioma & $178(29.1)$ & $97(32.3)$ & $81(26.0)$ & \\
\hline Astrocytoma & $171(28.0)$ & $110(36.7)$ & $61(19.6)$ & \\
\hline Glioblastoma & $145(23.7)$ & $19(6.3)$ & $126(40.2)$ & \\
\hline
\end{tabular}

\section{$\mathrm{B}, \mathrm{PFHH}$}

FOXL1

\begin{tabular}{|c|c|c|c|c|}
\hline \multirow[b]{2}{*}{ Variables } & \multirow[b]{2}{*}{ Cases, no. (\%) } & & \multirow[b]{2}{*}{ P-value } \\
\hline & & Low & High & \\
\hline Age (years) & & & & 0.075 \\
\hline$<60$ & $95(72.0)$ & $59(67.0)$ & $36(81.8)$ & \\
\hline$\geq 60$ & $37(28.0)$ & $29(33.0)$ & $8(18.2)$ & \\
\hline Sex & & & & 0.213 \\
\hline Female & $56(42.4)$ & $34(38.6)$ & $22(50.0)$ & \\
\hline Male & $76(57.6)$ & $54(61.4)$ & $22(50.0)$ & \\
\hline WHO grade & & & & 0.366 \\
\hline Low & $46(34.8)$ & $33(37.5)$ & $13(29.5)$ & \\
\hline High & $86(65.2)$ & $55(62.5)$ & $31(70.5)$ & \\
\hline Histological type & & & & 0.504 \\
\hline Oligoastrocytoma & $8(6.1)$ & $6(6.8)$ & $2(4.5)$ & \\
\hline Oligodendroglioma & $2(1.5)$ & $1(1.1)$ & $1(2.3)$ & \\
\hline Astrocytoma & $67(50.8)$ & $48(54.5)$ & $19(43.2)$ & \\
\hline Glioblastoma & $55(41.6)$ & $33(37.4)$ & $22(50.0)$ & \\
\hline
\end{tabular}

TCGA, The Cancer Genome Atlas; PFHH, patients from Huai'an First People's Hospital.

median: 19.6245, IQR: 21.27). In the PFHH cohort, expression of FOXL1 in tumor cells was detected by IHC. For further analysis, patients were divided into two groups with low expression of FOXL1 (IRS $\leq 6$ ) and high expression of FOXL1 (IRS >6) (Fig. 1).

Prognostic significance of FOXL1 expression in glioma patients. As shown in Fig. 2, in the TCGA cohort Kaplan-Meier analysis showed that WHO glioma grade $(\mathrm{P}<0.001$; Fig. $2 \mathrm{~A})$ and expression of FOXL1 $(\mathrm{P}<0.001$; Fig. 2B) were associated with OS. We further made subgroup analyses of FOXL1 expression according to patients' WHO grade. The results indicated that FOXL1 expression correlated with OS in high grade subgroup $(\mathrm{P}<0.001$; Fig. 2D), while low grade subgroup had no such correlation $(\mathrm{P}=0.250$; Fig. $2 \mathrm{C})$. The univariate Cox regression model revealed that age $(\mathrm{P}<0.001)$, WHO grade $(\mathrm{P}<0.001)$, histological type $(\mathrm{P}<0.001)$ and FOXL1 expression $(\mathrm{P}<0.001)$ were associated with prognosis 

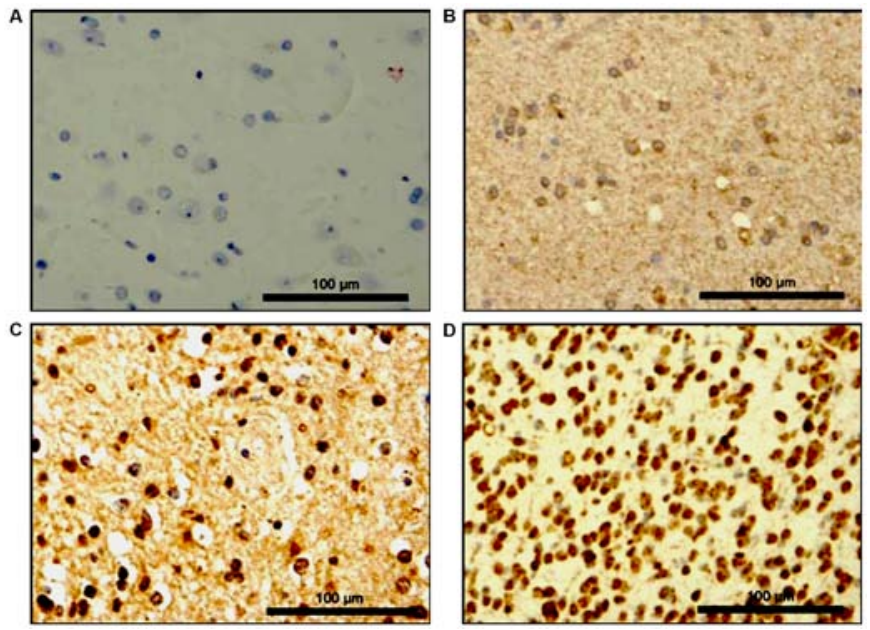

Figure 1. IHC staining characteristics of FOXL1. (A) Absent FOXL1 expression; (B) weak staining of FOXL1; (C) moderate staining of FOXL1; (D) strong staining of FOXL1. (A-D) Magnification, x200. IHC, immunohistochemistry.
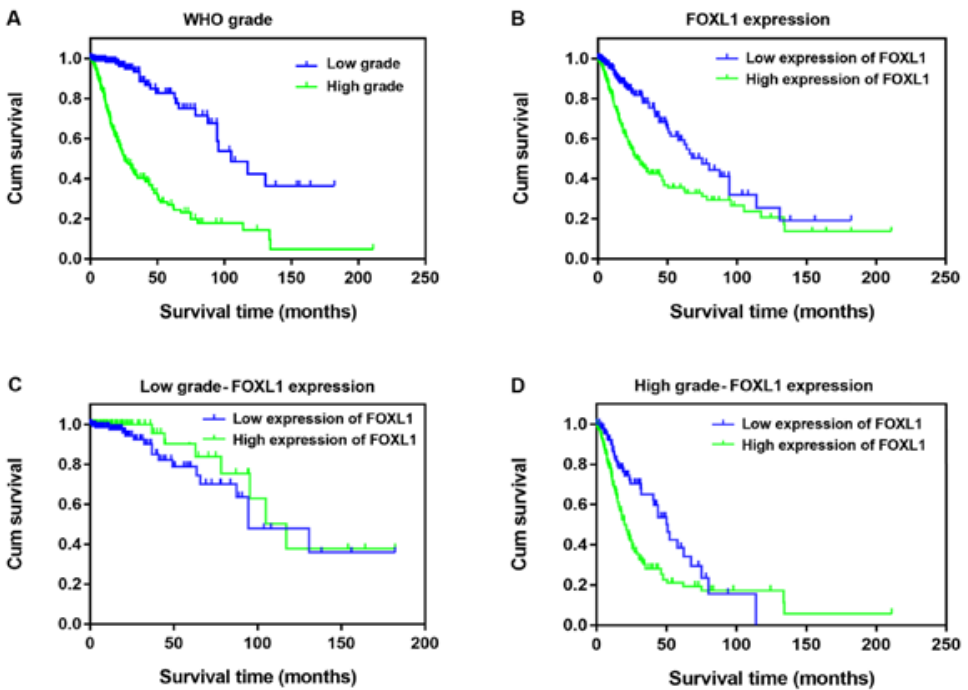

Figure 2. Survival analysis using Kaplan Meier method in TCGA cohort. (A) WHO grade ( $<<0.001)$; (B) FOXL1 expression (P<0.001); (C and D) subgroup analyses for FOXL1 expression in low grade $(\mathrm{P}=0.250)$ and high grade $(\mathrm{P}<0.001)$ glioma. TCGA, The Cancer Genome Atlas.

A
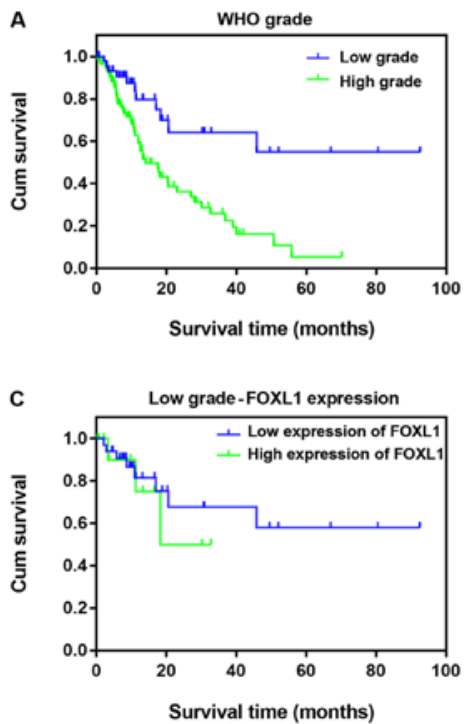

B

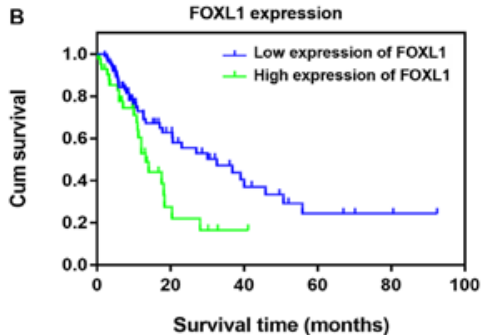

D

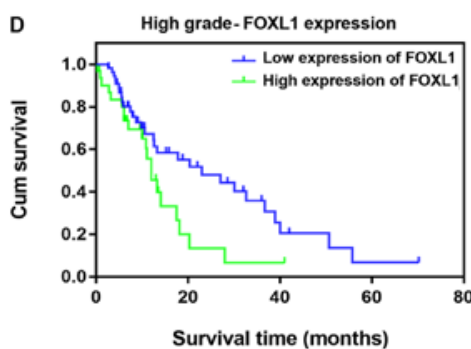

Figure 3. Survival analysis using Kaplan Meier method in PFHH cohort. (A) WHO grade ( $\mathrm{P}<0.001)$; (B) FOXL1 expression ( $\mathrm{P}=0.012)$; (C and D) subgroup analyses for FOXL1 expression in low grade $(\mathrm{P}=0.585)$ and high grade $(\mathrm{P}=0.034)$ glioma. $\mathrm{PFHH}$, patients from Huai'an First People's Hospital 
Table II. Univariate and multivariate Cox regression analysis of overall survival.

A, TCGA

\begin{tabular}{|c|c|c|c|c|c|c|}
\hline \multirow[b]{2}{*}{ Variables } & \multicolumn{3}{|c|}{ Univariate analysis } & \multicolumn{3}{|c|}{ Multivariate analysis } \\
\hline & HR & $95 \% \mathrm{CI}$ & $\mathrm{P}$-value & HR & $95 \% \mathrm{CI}$ & P-value \\
\hline Age & & & $<0.001$ & & & $<0.001$ \\
\hline$<60$ & 1 & & & & & \\
\hline$\geq 60$ & 4.983 & 3.644-6.814 & & 2.504 & $1.787-3.507$ & \\
\hline Sex & & & 0.629 & & & \\
\hline Female & 1 & & & & & \\
\hline Male & 1.076 & $0.800-1.446$ & & & & \\
\hline WHO grade & & & $<0.001$ & & & $<0.001$ \\
\hline Low & 1 & & & & & \\
\hline High & 6.007 & $3.891-9.274$ & & 2.958 & $1.831-4.778$ & \\
\hline Histological type & & & $<0.001$ & & & $<0.001$ \\
\hline Oligoastrocytoma & 1 & & & & & \\
\hline Other types & 2.752 & $2.293-3.303$ & & 1.823 & $1.505-2.208$ & \\
\hline FOXL1 & & & $<0.001$ & & & 0.281 \\
\hline Low & 1 & & & & & \\
\hline High & 2.274 & $1.661-3.113$ & & 1.203 & $0.860-1.683$ & \\
\hline
\end{tabular}

\section{$\mathrm{B}, \mathrm{PFHH}$}

\begin{tabular}{|c|c|c|c|c|c|c|}
\hline Variables & HR & $95 \% \mathrm{CI}$ & P-value & $\mathrm{HR}$ & $95 \% \mathrm{CI}$ & P-value \\
\hline Age & & & 0.571 & & & \\
\hline$<60$ & 1 & & & & & \\
\hline$\geq 60$ & 1.172 & $0.677-2.028$ & & & & \\
\hline Sex & & & 0.793 & & & \\
\hline Female & 1 & & & & & \\
\hline Male & 1.072 & $0.637-1.805$ & & & & \\
\hline WHO grade & & & 0.001 & & & 0.040 \\
\hline Low & 1 & & & & & \\
\hline High & 3.068 & $1.585-5.940$ & & 2.177 & $1.036-4.574$ & \\
\hline Histological type & & & $<0.001$ & & & 0.027 \\
\hline Oligoastrocytoma & 1 & & & & & \\
\hline Other types & 2.396 & $1.487-3.860$ & & 1.688 & $1.063-2.680$ & \\
\hline FOXL1 & & & 0.013 & & & 0.054 \\
\hline Low & 1 & & & & & \\
\hline High & 1.981 & $1.152-3.406$ & & 1.710 & $0.990-2.952$ & \\
\hline
\end{tabular}

TCGA, The Cancer Genome Atlas; PFHH, patients from Huai'an First People's Hospital.

of glioma patients in terms of OS in the TCGA cohort. Multivariate Cox regression after adjustment indicated that age $(\mathrm{P}<0.001)$, and WHO grade $(\mathrm{P}<0.001)$, histological type $(\mathrm{P}<0.001)$ were independent prognostic factors for OS in glioma patients and FOXL1 expression lost its significance $(\mathrm{P}=0.281$; Table II).

In the PFHH cohort, using Kaplan-Meier analysis it was found that WHO glioma grade $(\mathrm{P}<0.001$; Fig. $3 \mathrm{~A})$ and expression of FOXL1 ( $\mathrm{P}=0.012$; Fig. $3 \mathrm{~B})$ were associated with OS in glioma patients. Subgroup analyses showed that FOXL1 expression correlated with OS in high grade subgroup $(\mathrm{P}=0.034$; Fig. 2D). The univariate Cox regression showed that WHO grade $(\mathrm{P}=0.001)$, histological type $(\mathrm{P}<0.001)$ and FOXL1 expression $(\mathrm{P}=0.013)$ were associated with prognosis of glioma patients in the PFHH cohort. After adjustment multivariate Cox regression revealed that $\mathrm{WHO}$ grade $(\mathrm{P}=0.040)$ and histological type $(\mathrm{P}=0.027)$ were independent prognostic factors for OS in glioma patients. 


\section{Discussion}

Previous studies have suggested that FOXL1, a critical transcription factor, plays an important role in regulation of cell proliferation and development of the epithelium in gastrointestinal tracts in mice $(20,21)$. The roles of FOXL1 in gastrointestinal cancers have been widely investigated (12-14). Furthermore, FOXL1 has been reported to be associated with regulation of central nervous system development in zebrafish (16), suggesting that FOXL1 may also have an effect on brain cancers. However, limited evidence is available on the role of FOXL1 in brain cancers. To the best of our knowledge, this is one of the first studies to demonstrate the associations of FOXL1 with clinicopathological features in glioma patients. Our data showed for the first time that a higher FOXL1 expression is associated with worse clinical outcome in glioma patients from TCGA and PFHH cohorts. These findings suggested that FOXL1 may be involved in tumorigenesis and progression of glioma and may serve as a candidate predictor of clinical outcome in glioma patients undergoing surgery.

Downregulation of FOXL1 has been studied in several malignant tumors. In a mechanistic research using ApcMin mice, Perreault et al demonstrated that FOXL1 was the first mesenchymal modifier of Min and plays a key role in gastrointestinal tumorigenesis (12). In another study, Zhang et al reported that FOXL1, as a novel tumor suppressor candidate, could inhibit tumor aggressiveness and predict better clinical outcome in human pancreatic cancer (13). They also revealed that FOXL1 promoted apoptosis was partly through the induction of TNF-related apoptosis-inducing ligand (TRAIL) in pancreatic cancer cells (13). Qin et al reported that FOXL1 could also suppress tumorigenicity in gallbladder cancer. The underlying mechanism may refer to the disruption of mitochondrial transmembrane potential and triggering mitochondria-mediated apoptosis (10). However, the mechanisms for downexpression of FOXL1 in these cancers have not been fully elucidated yet. In addition, some other mechanisms were also proposed to be associated with deregulation of Fox factors, including chromosome translocations $(22,23)$, chromosomal deletion (24), promoter methylation (25), alteration in upstream regulators $(26,27)$ and post-translational modifications. Further functional studies referring to FOXL1 downregulation in cancers are still needed.

It is interesting to note that the results presented here show that higher FOXL1 expression in gliomas is associated with a worse clinical outcome, which is different from other malignant tumors. However, limited information is available on the underlying mechanism concerning the observations. One study using zebra fish reported that FOXL1 was strongly expressed in neural tissues (16). This result may indicate that FOXL1 expression pattern in neural tissues is different from other tissues, which may give a hint to the future investigations. Further studies on genetic and epigenetic mechanisms ought to be carried out to clarify the issue.

There are some limitations that should be considered in our study. Although we have analyzed a large cohort, the present study is a retrospective analysis and there is a potential or selection bias. Additionally, the relatively small sample of histological subgroups in PFHH cohort may result in lack of statistical power for the Cox regression analysis.

In conclusion, this study showed that higher expression of FOXL1 is associated with worse outcome of glioma patients in TCGA and PFHH cohorts. Especially, FOXL1 expression is associated with OS in high grade subgroup. Our findings suggest that FOXL1 expression is a candidate predictor of clinical outcome in glioma patients and may act as an effective molecular marker for immunotherapeutic strategies of glioma patients in clinical practice.

\section{Acknowledgements}

We would like to thank Xiangyang Tian and Hua Cao for helpful suggestions during the preparation of the manuscript.

\section{Funding}

No funding was received.

\section{Availability of data and material}

The datasets used and/or analyzed during the current study are available from the corresponding author on reasonable request.

\section{Authors' contributions}

$\mathrm{AC}$ wrote the manuscript and helped with immunohistochemical staining. LZ and JL collected and analyzed general information of patients and contributed to statistical analysis. All authors read and approved the final manuscript.

\section{Ethics approval and consent to participate}

This study was approved by the Ethics and Research Committees of Huai'an First People's Hospital (Huai'an, China), (Project identification code: IRB-KPJ2017-003-01). Patients who participated in this research had complete clinical data. The signed informed consents were obtained from the patients or the guardians.

\section{Patient consent for publication}

Not applicable.

\section{Competing interests}

The authors declare that they have no competing interests.

\section{References}

1. Goodenberger ML and Jenkins RB: Genetics of adult glioma. Cancer Genet 205: 613-621, 2012.

2. Louis DN, Ohgaki H, Wiestler OD, Cavenee WK, Burger PC, Jouvet A, Scheithauer BW and Kleihues P: The 2007 WHO classification of tumours of the central nervous system. Acta Neuropathol 114: 97-109, 2007.

3. Cheng YS, Lin C, Cheng YP, Yu YL, Tang CT and Hueng DY: Epithelial cell transformation sequence 2 is a potential biomarker of unfavorable survival in human gliomas. Neurol India 62: 406-409, 2014 
4. Stupp R, Mason WP, van den Bent MJ, Weller M, Fisher B, Taphoorn MJ, Belanger K, Brandes AA, Marosi C, Bogdahn U, et al; European Organisation for Research and Treatment of Cancer Brain Tumor and Radiotherapy Groups National Cancer Institute of Canada Clinical Trials Group: Radiotherapy plus concomitant and adjuvant temozolomide for glioblastoma. N Engl J Med 352: 987-996, 2005.

5. Bell C, Dowson N, Fay M, Thomas P, Puttick S, Gal Y and Rose S: Hypoxia imaging in gliomas with $18 \mathrm{~F}$-fluoromisonidazole PET: Toward clinical translation. Semin Nucl Med 45: 136-150, 2015.

6. Lam EW, Brosens JJ, Gomes AR and Koo CY: Forkhead box proteins: tuning forks for transcriptional harmony. Nat Rev Cancer 13: 482-495, 2013.

7. Lehmann OJ, Sowden JC, Carlsson P, Jordan T and Bhattacharya SS: Fox's in development and disease. Trends Genet 19: 339-344, 2003.

8. Carter ME and Brunet A: FOXO transcription factors. Curr Biol 17: R113-R114, 2007.

9. Wang Z, Ahmad A, Li Y, Banerjee S, Kong D and Sarkar FH: Forkhead box M1 transcription factor: a novel target for cancer therapy. Cancer Treat Rev 36: 151-156, 2010.

10. Qin Y, Gong W, Zhang M, Wang J, Tang Z and Quan Z: Forkhead box L1 is frequently downregulated in gallbladder cancer and inhibits cell growth through apoptosis induction by mitochondrial dysfunction. PLoS One 9: e102084, 2014.

11. Takano-Maruyama M, Hase K, Fukamachi H, Kato Y, Koseki H and Ohno H: Foxl1-deficient mice exhibit aberrant epithelial cell positioning resulting from dysregulated EphB/EphrinB expression in the small intestine. Am J Physiol Gastrointest Liver Physiol 291: G163-G170, 2006.

12. Perreault N, Sackett SD, Katz JP, Furth EE and Kaestner KH: Foxl1 is a mesenchymal modifier of Min in carcinogenesis of stomach and colon. Genes Dev 19: 311-315, 2005.

13. Zhang G, He P, Gaedcke J, Ghadimi BM, Ried T, Yfantis HG, Lee DH, Hanna N, Alexander HR and Hussain SP: FOXL1, a novel candidate tumor suppressor, inhibits tumor aggressiveness and predicts outcome in human pancreatic cancer. Cancer Res 73: 5416-5425, 2013.

14. Ertao Z, Jianhui C, Chuangqi C, Changjiang Q, Sile C, Yulong $\mathrm{H}$, Shirong $\mathrm{C}$ and Hui W: Low level of FOXL1 indicates a worse prognosis for gastric cancer patients. Tumour Biol 37: 11331-11337, 2016.

15. Yang FQ, Yang FP, Li W, Liu M, Wang GC, Che JP, Huang JH and Zheng JH: Foxl1 inhibits tumor invasion and predicts outcome in human renal cancer. Int J Clin Exp Pathol 7: 110-122, 2013.

16. Nakada C, Satoh S, Tabata Y, Arai K and Watanabe S: Transcriptional repressor foxl1 regulates central nervous system development by suppressing shh expression in zebra fish. Mol Cell Biol 26: 7246-7257, 2006.

17. Jiang YZ, Yu KD, Zuo WJ, Peng WT and Shao ZM: GATA3 mutations define a unique subtype of luminal-like breast cancer with improved survival. Cancer 120: 1329-1337, 2014.
18. Li Y, Liang L, Dai W, Cai G, Xu Y, Li X, Li Q and Cai S: Prognostic impact of programed cell death-1 (PD-1) and PD-ligand 1 (PD-L1) expression in cancer cells and tumor infiltrating lymphocytes in colorectal cancer. Mol Cancer 15: 55, 2016.

19. Ueno H, Jones AM, Wilkinson KH, Jass JR and Talbot IC: Histological categorisation of fibrotic cancer stroma in advanced rectal cancer. Gut 53: 581-586, 2004.

20. Kaestner KH, Silberg DG, Traber PG and Schütz G: The mesenchymal winged helix transcription factor Fkh6 is required for the control of gastrointestinal proliferation and differentiation. Genes Dev 11: 1583-1595, 1997.

21. Kaestner KH, Bleckmann SC, Monaghan AP, Schlöndorff J, Mincheva A, Lichter P and Schütz G: Clustered arrangement of winged helix genes fkh-6 and MFH-1: possible implications for mesoderm development. Development 122: 1751-1758, 1996.

22. Wlodarska I, Veyt E, De Paepe P, Vandenberghe P, Nooijen P, Theate I, Michaux L, Sagaert X, Marynen P, Hagemeijer A, et al: FOXP1, a gene highly expressed in a subset of diffuse large B-cell lymphoma, is recurrently targeted by genomic aberrations. Leukemia 19: 1299-1305, 2005.

23. Streubel B, Vinatzer U, Lamprecht A, Raderer $M$ and Chott A: $\mathrm{T}(3 ; 14)(\mathrm{p} 14.1 ; \mathrm{q} 32)$ involving IGH and FOXP1 is a novel recurrent chromosomal aberration in MALT lymphoma. Leukemia 19: 652-658, 2005

24. Dong XY, Chen C, Sun X, Guo P, Vessella RL, Wang RX, Chung LW, Zhou W and Dong JT: FOXO1A is a candidate for the 13q14 tumor suppressor gene inhibiting androgen receptor signaling in prostate cancer. Cancer Res 66: 6998-7006, 2006.

25. Halmos B, Bassères DS, Monti S, D'Aló F, Dayaram T, Ferenczi K, Wouters BJ, Huettner CS, Golub TR and Tenen DG: A transcriptional profiling study of CCAAT/enhancer binding protein targets identifies hepatocyte nuclear factor 3 beta as a novel tumor suppressor in lung cancer. Cancer Res 64: 4137-4147, 2004.

26. Brunet A, Bonni A, Zigmond MJ, Lin MZ, Juo P, Hu LS, Anderson MJ, Arden KC, Blenis J and Greenberg ME: Akt promotes cell survival by phosphorylating and inhibiting a Forkhead transcription factor. Cell 96: 857-868, 1999.

27. Hu MC, Lee DF, Xia W, Golfman LS, Ou-Yang F, Yang JY, Zou Y, Bao S, Hanada N, Saso H, et al: IkappaB kinase promotes tumorigenesis through inhibition of forkhead FOXO3a. Cell 117: 225-237, 2004. 